\title{
A protocol for evidence-based targeting and evaluation of statewide strategies for preventing falls among community-dwelling older people in Victoria, Australia
}

\author{
Lesley Day, ${ }^{1}$ Caroline F Finch, ${ }^{1}$ Keith D Hill, ${ }^{2,3,4}$ Terry P Haines, ${ }^{1,5}$ Lindy Clemson, ${ }^{6}$ \\ Margaret Thomas, ${ }^{7}$ Catherine Thompson ${ }^{7}$
}

${ }^{1}$ Monash University, Melbourne, Australia

${ }^{2}$ Latrobe University, Melbourne, Australia

${ }^{3}$ Northern Health, Melbourne,

Australia

${ }^{4}$ National Ageing Research Institute, Melbourne, Australia

${ }^{5}$ Southern Health, Melbourne, Australia

${ }^{6}$ University of Sydney, Sydney, Australia

${ }^{7}$ Victorian Department of Health, Melbourne, Australia

\section{Correspondence to}

Dr Lesley Day, Accident Research Centre, Monash University, Clayton, Melbourne, Victoria 3800, Australia; lesley.day@monash.edu

Accepted 1 December 2010 Published Online First 24 December 2010

\section{UNLOCKA}

This paper is freely available online under the BMJ Journals unlocked scheme, see http:// injuryprevention.bmj.com/site/ about/unlocked.xhtml

\section{ABSTRACT}

Background Falls are a significant threat to the safety, health and independence of older citizens. Despite the now substantial evidence about effective falls prevention interventions, translation into falls reductions has not yet been fully realised. While the hip fracture rate is decreasing, the number and rate of fall-related hospital admissions among older people is increasing. The challenge now is to deliver the most effective interventions efficiently at a population level, and for these interventions to be taken up by older people. Objective To support the development, and evaluation of, effective falls prevention policy and practice in the state of Victoria, Australia.

Methods The RE-AIM model (Reach, Efficacy, Adoption, Implementation, Maintenance) was used to identify strategies for an effective programme. Research objectives were developed to support the strategies. These include: (1) identification of subgroups of older people most frequently admitted to hospital for falls; (2) examining the acceptability of established falls interventions; (3) identification of factors that encourage and support relevant lifestyle changes; (4) identifying opportunities to incorporate confirmed interventions in existing programmes and services; (5) developing guidelines for sustainability. The research results will subsequently guide strategy details for the falls prevention plan. RE-AIM will provide the framework for the evaluation structure.

Outcome measures Measures to monitor the implementation of the selected interventions will be determined for each intervention, based on the five key factors of the RE-AIM model. The overall effect of the falls prevention plan will be monitored by time series analysis of fall-related hospital admission rates for community-dwelling older people.

Falls are a significant threat to the safety, health and independence of Australia's older citizens, accounting for $62 \%$ of accidental deaths for people over 75 years, ${ }^{1}$ and at least $1 \%$ and $4 \%$ of all hospital separations for older men and women, respectively. ${ }^{2}{ }^{3}$ Falls are relatively common, with $28-39 \%$ of people aged over 65 years experiencing at least one fall annually, and up to $50 \%$ experiencing multiple falls. ${ }^{4}{ }^{5}$ A constellation of adverse health outcomes can follow. Approximately $20 \%$ of all fall incidents among community-dwelling older people require medical attention, and $10 \%$ result in serious injury. ${ }^{67} \mathrm{~A}$ hip fracture has far-reaching effects on an individual, their friends and family and the community, and possibly contributes to an increased mortality risk. ${ }^{3}$ Other equally significant outcomes include erosion of overall health status, confidence, mobility and independence, contributing to decreased quality of life and often prompting the move to residential aged care. ${ }^{8-11}$

Population ageing is one of the major transformations being experienced in Australia. The population aged over 65 years is projected to increase from $13 \%$ in 2008 to $24-25 \%$ by 2056 , with the number of people over 80 years expanding most substantially. ${ }^{12}$ Health gains that may occur as the more active and better educated "baby boomers' age and have access to improved disease management are likely to be offset by an expected increase in disability for those over 80 years. ${ }^{13}$ Falls prevention is an important component of promoting healthy and independent ageing, and containing medical and support service costs. The total cost of fall-related injury is expected to rise dramatically, tripling to $\mathrm{A} \$ 1375$ million per year, over the next few decades unless effective prevention and lower treatment costs occur. ${ }^{14}$ There is already some evidence of an increasing fall-related burden on the health system. ${ }^{15-17}$ Furthermore, recent work suggests that the earlier cost projections are underestimates. ${ }^{14}$

There is now substantial evidence about effective falls prevention interventions, particularly among community-dwelling older people, synthesised in a Cochrane Review. ${ }^{7}$ The recommended interventions include: tai chi group exercise; group exercise containing multi-components; individually prescribed home-based muscle strengthening and balance training; home hazard assessment and modification that is professionally prescribed, particularly for older people with a history of falling; reduction of psychotropic medication; medication review by primary care physicians; multidisciplinary multifactorial risk factor screening and intervention programmes; first eye cataract surgery; vitamin D supplementation for those with deficiency; and cardiac pacing for fallers with cardioinhibitory carotid sinus hypersensitivity. However, the potential for the research evidence base to translate into falls reductions has not yet been fully realised. The challenge now is to deliver the most effective interventions efficiently at a population level, and for these interventions to be taken up by older people. ${ }^{18-22}$ 
Victoria is the second most populous state in Australia, with a population of 5.496 million. The Victorian government is committed to improving the health and wellbeing of all Victorians and recognises that maintaining an active and healthy lifestyle ensures wellbeing and independence in older age. The Department of Health (the department) covers the responsibilities of the Ministers for Senior Victorians, Health and Mental Health, Community Services and Housing. The Aged Care Branch (ACB) has lead policy responsibility for falls prevention among older people in Victoria. The ACB works actively with the National Injury Prevention Working Group on national issues such as implementing national guidelines in all settings, engaging general practice and workforce development. Since 1998, the ACB has invested an average of A $\$ 1.25$ million per year in falls prevention interventions in Victoria. ACB projects incorporate a multi-intervention approach and are typically implemented within departmental programmes and structures, such as primary care partnerships (PCP) and integrated health promotion, as 'add-on' interventions delivered with specific fixed project funding. The department has also supported a falls prevention practitioners' network by hosting network meetings and working subgroups, thereby providing an avenue for practitioners to influence the service system and policy context for falls. ${ }^{23}$

Although there is evidence that the hospital admission rate for hip fracture in Victoria has decreased, both the numbers and rate for all falls-related hospital admissions among older people have been increasing. ${ }^{15}$ This indicates that a new approach is needed if the predicted health system burden due to falls is to be moderated. The department wants to facilitate better targeting of available falls prevention investment and will use this partnership project to generate and integrate evidence to support a reorientation of the current approach. This protocol describes elements of a new direction, which include: consideration of older people's preferences for different interventions; identification of those subgroups of older people who use the greater proportion of inpatient healthcare services; consideration of factors that will encourage intervention uptake; and identification of strategies to enhance intervention sustainability beyond any initial injection of project-based funding. Furthermore, the department has responsibility for a range of services likely to be relevant to falls prevention, including: acute healthcare (hospital inpatient and outpatient services); specialist services (falls clinics); primary healthcare; and community care, some of which have not yet been considered as potential vehicles for the delivery of falls prevention interventions.

Therefore, the aim of this falls prevention partnership is to enable a more effective policy response to the falls prevention challenge in Victoria. The proposed research will underpin a reorientation of the Department of Health falls prevention programme and evaluate its delivery.

The RE-AIM model has been chosen to underpin this proposal because it is compatible with contemporary systems-based thinking and population health approaches. ${ }^{24}$ The model posits that the impact of a public health intervention is a function of five factors: reach, efficacy, adoption, implementation and maintenance. Therefore, these five factors should be central to prevention policy and programme planning, as well as forming the framework for programme evaluation. Our proposed research programme provides and integrates the evidence required to inform falls prevention policy and programme planning within the RE-AIM framework.

\section{RESEARCH PLAN}

The research plan comprises a series of discrete components that will be integrated through the RE-AIM model into a 2-year falls prevention programme plan for Victoria. The objectives are to:

1. Facilitate improved targeting of evidence-based falls interventions to groups of older people most frequently hospitalised for fall-related conditions.

2. Identify factors that encourage and support older people to make relevant fall-preventing lifestyle changes.

3. Examine the acceptability of the currently confirmed falls interventions to older people.

4. Identify opportunities in existing programmes and services to incorporate evidence-based falls interventions into policies and practices, especially when there is a good match between current priorities and the established falls interventions.

5. Develop and test guidelines for sustainability based on the known factors that encourage and support relevant agencies and programmes to incorporate and sustain falls interventions. These objectives were identified following an analysis of the current state of knowledge relative to the requirements indicated by the RE-AIM model (table 1). The objectives will be achieved through four research components that are outlined below with a short rationale and methods.

\section{Research component 1: identifying priority groups for improved targeting of falls interventions \\ Rationale}

Ongoing policy development and implementation requires information on the health status of individuals targeted by that policy. $^{25}$ Australian studies have highlighted the continuing significant burden of, and trends associated with, hospitalised falls in older people. ${ }^{2}{ }^{15-17}$ Moreover, there is a high rate of hospitalisation for people with major types of falls injury (notably hip and pelvic fracture) presenting to Victorian emergency departments. ${ }^{26}$ While these broad scale epidemiological studies have been critical for demonstrating the need for policy, systemic and practice responses to a problem of this magnitude and forecasted trends, these types of studies have not identified specific subgroups of older people (other than age and gender) who should be the target for falls interventions. An example of such a subgroup might be those who use the most bed days for hospitalised falls, and thus who have the greatest impact on healthcare costs. Preliminary analysis of Victorian hospitalisations data indicates that $67 \%$ of older admitted fallers have at least one co-morbid condition, with heart disease (37\%) and diabetes $(25 \%)$ being the most prevalent. Unpublished data for the state

Table 1 RE-AIM framework

\begin{tabular}{lll}
\hline RE-AIM factor & Planning dimension* & Evaluation dimension \\
\hline Reach & Strategies to target population $(\mathrm{RC} 1,2)$ & Extent to which target population participated \\
Efficacy & Evidence for intervention effectiveness (published evidence) & Net impact on targeted health or risk indicator \\
Adoption & Strategies to develop the required organisational support $(\mathrm{RC} 3,4)$ & Extent to which target settings or organisations adopt policy or intervention \\
Implementation & Strategies to ensure delivery as intended $(\mathrm{RC}, 4)$ & Extent to which intervention is delivered as intended \\
Maintenance & Strategies to enhance sustainability $(\mathrm{RC}, 4)$ & Extent that individuals and organisations maintain behaviour and/or policy change
\end{tabular}

*The research component (RC) supporting each planning dimension is shown in brackets. 
New South Wales show that the higher rate of mortality following falls hospitalisation in men can be accounted for by co-morbidities, consistent with a recent Italian study. ${ }^{27}$ Little research has been undertaken to identify the relationship between co-morbid conditions and falls injury risk or outcomes, and most of this has only examined mortality. Given the high proportion of hospitalised fallers with co-morbidities, addressing this major gap is likely to identify new falls prevention opportunities and specifically target subgroups of older people.

\section{Methods}

The Victorian Admitted Episodes Database will be used to identify the characteristics and co-morbidity profiles of older people admitted to hospital following a fall, particularly those using more bed days. Hospital admissions with an external cause code are routinely extracted from the Victorian Admitted Episodes Database by the Victorian Department of Health and supplied in unit record format to the Victorian Injury Surveillance Unit (at the Monash University Accident Research Centre) every 6 months. For this project, cases of fall-related hospitalisations for over 65 year olds will be defined by International Classification of Diseases, version 10 AM external cause of injury codes W00-W19. Standardised approaches for accounting for repeat admissions for the same fall injury will be adopted. ${ }^{15} 28$ For each case, the following characteristics will be extracted and analysed: age, gender, marital status, country of birth, Barthel index on admission and discharge, discharge status, local government area of usual residence, type of residence before admission and on discharge, insurance status, number and nature of the co-morbid conditions and type of injury sustained.

Reported co-morbidities will be categorised with the Charlson comorbidity index (CCI), a weighted index that takes account of both the number and seriousness of co-morbid diseases. ${ }^{29} \mathrm{~A} \mathrm{CCI}$ of 1-2 will be classed as 'mild' co-morbidity, and a CCI of 3 or greater will indicate 'more severe' co-morbidity. The analyses will also be categorised according to the International Classification of Diseases, version 10 AM 'place of occurrence' codes, which will be used to identify falls that occur in specific settings such as private dwellings, residential facilities and public places. The local government area data will be used to determine differences across the state according to select sociodemographic profiles using methods and approaches used previously. ${ }^{30} 31$

Cross tabulations of patient characteristics by total bed days and the CCI will be prepared. Negative binomial regression models $^{32}$ will be applied to describe and understand the relationships between the variables and to identify those characteristics most associated with high bed day use and high CCI. Predictive models will be developed through generalised estimating equations to identify groups, or clusters, of patients with specific related characteristics and outcomes. In year 1 , these analyses will focus on the most recently available data (2006/7). In subsequent years, the models will be updated and refined if necessary. The predictive power of the first model will be assessed against subsequent data, to ensure that the clusters identified for intervention targeting remain robust. Results from this component will inform all other research components and the falls prevention plan.

\section{Research component 2: identifying acceptability of falls interventions and likely barriers to, and facilitators for, individual action \\ Rationale}

Cost-effective falls interventions need to be adopted by older people if the potential for falls reductions is to be maximised.
Consensus recommendations for promoting the uptake and adherence to falls-related interventions have been developed in Europe. ${ }^{19}$ The recommendations were based on available research (much of it undertaken in Europe), and were informed by basic psychosocial theory and expert opinion. Although clear directions were identified, the authors noted the limited evidence base underpinning these recommendations and stressed the need for confirmative studies such as those proposed in this project.

Research into the acceptability and uptake of established falls prevention measures among older people is even more limited in Australia. The strong theme regarding improving health and maintaining independence as a motivator for action rather than falls prevention apparent in the European study ${ }^{33}$ was also observed in two small Australian qualitative studies. ${ }^{34} 35$ The preference for home safety modifications and reluctance to attend group exercise sessions observed in the $\mathrm{UK}^{20}$ had also been reported among 60 older fallers presenting to a hospital emergency department in South Australia. ${ }^{36}$ Reluctance to undertake group-based exercise programmes was not found in another Australian study. ${ }^{37}$ However, this survey had a low response rate $(23 \%)$, and comprised physically active people, confident of their ability to prevent falling.

The acceptance of, and likely uptake of, confirmed falls interventions is clearly an important factor in the success of falls prevention programmes. Given the lack of quantitative data in the Australian context, establishing likely adoption, and the circumstances in which this could be maximised, is a critical step underpinning the falls prevention plan. The aims of this component are to: identify the leading falls interventions likely to be undertaken by older adults; identify factors that strongly influence the intention to undertake leading falls interventions so that falls interventions can be delivered in a manner that will maximise adoption.

\section{Methods}

A cross-sectional telephone survey of the priority participant subgroups identified from component 1 will be undertaken. Based on preliminary data, it is likely that community-dwelling older people, particularly women aged over 75 years, and those with co-morbid conditions, will emerge as priority groups. The proposed methods are described for these groups to illustrate our approach. If different subgroups emerge as a priority, then recruitment methods will be adjusted as necessary. The theoretical framework for the survey (figure 1) draws primarily upon the health belief model, ${ }^{38}$ with additional input from other relevant theories. ${ }^{38}{ }^{39}$ Central to this framework is the individual's weighing up of anticipated benefits from undertaking an intervention as opposed to anticipated costs and disbenefits, to form an intention to undertake the intervention. Previous work using a similar framework found factors related to coping (theory of planned behaviour) were most strongly associated with the intention to undertake community-based strength and balance training among community-dwelling older adults. ${ }^{40} \mathrm{~A}$ limitation of this study was that the description of strength and balance training provided to participants was a heterogenous collection of activities with little description of how and where the exercise would take place.

The survey instrument will be constructed using one or two five-point Likert scale items (strongly agree to strongly disagree with undecided as a central point) for each domain of the framework (figure 1). The influence of health practitioner recommendation, a likely contributor to several domains will also be included. Examples of questions include: self efficacy-'I am confident that I can (perform the intervention)'; intention to 
Figure 1 Theoretical framework for understanding uptake of falls prevention interventions.

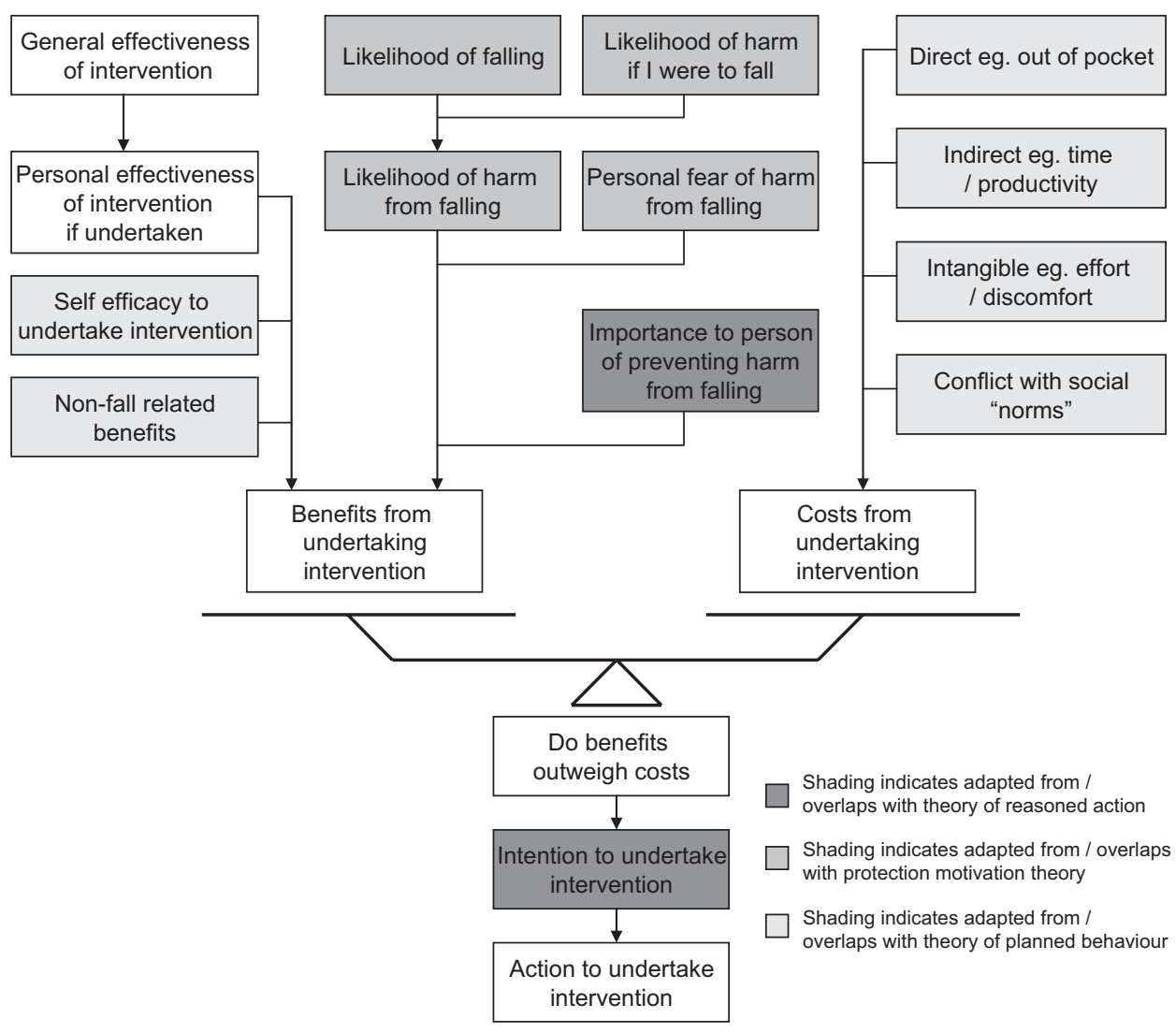

undertake intervention-'I intend to (perform the activity)'; social norms 'It is normal for people like me to (perform the activity)' and health practitioner influence 'I would (perform the activity) if my doctor recommended it'. Questions will be developed for the most promising falls interventions for the subgroups identified in component 1 as determined by the Cochrane review and subsequent modelling undertaken by the applicants. ${ }^{7} 18$ The selected interventions, the likely location, time of day and out-of-pocket expenses will be described. Visual cues showing people participating in the interventions will be used. Item sets related to each of the interventions will be presented in a randomly determined sequence to account for a potential order effect. Additional explanatory variables will be collected, including: participant demographics; falls history; selfidentified risk factors for falls; medical conditions; previous exposure to the intervention; household income; access to transport; social support; independence in activities of daily living.

This survey builds on previous work examining attitudes to participation in inhospital falls interventions. ${ }^{41}$ In that study, high self-efficacy to attempt a self-identified falls intervention, and belief that the intervention reduces falls risk, were both correlated moderately with reported intent $(\rho=0.57,0.60$, respectively, $\mathrm{p}<0.001)$.

The sampling frame will be the representative state-wide sample recruited for the Victorian Population Health Household Survey, undertaken annually by the department, in which respondents are asked for permission to be re-contacted for future research. Consenting community-dwelling people over 75 years $(n=500)$ will be re-contacted for recruitment to this survey. The sample will be constructed to comprise equal numbers of women over 75 years and men and women over 65 years with at least one of the more prevalent co-morbid conditions identified in component 1 . While there will be some overlap between the eligibility criteria for these two subgroups, sufficient power will be required to analyse the data for those with co-morbid conditions as a discrete group.

The 'intention to participate' item will be examined in pairwise comparisons between the interventions using the Wilcoxon signed-ranks test, while associations with covariates will be examined using ordinal logistic regression. The survey sample sizes have been determined on the basis of the Wilcoxon signed-ranks test and should ensure $90 \%$ power to detect a 'shift' effect size as low as 0.2 for each of the two different participant groups. ${ }^{42}$

\section{Research component 3: opportunities to integrate evidence-based falls interventions into departmental policies and programmes \\ Rationale}

Victorian state government departments including health, human services and planning and community development, deliver a wide range of health, community and aged care and housing services. Given the nature of the established falls interventions (eg, exercise programmes, home safety assessments, multifactorial risk factor assessments), and the fact that many community-dwelling older people are reached each year by departmental services, it would seem that many opportunities would exist to integrate falls interventions into departmental policies and programmes. Indeed, the ACB has identified some relevant programmes and has made some progress towards the delivery of falls interventions in parallel with other services. However, a comprehensive stock-take has not been undertaken, new opportunities continue to arise and need to be captured, and sustainable integration is elusive.

\section{Methods}

Opportunities for the incorporation of evidence-based falls interventions into the policies and programmes will be identified using a stepwise approach. The methods will be 
modelled on our previous policy influence work. ${ }^{18} 4344$ The first step (step 1) will be to identify the broad range of department polices and programmes in which there may be scope for integration. A meeting of senior departmental managers (eg, from rural and regional health and aged care services division branches: aged care, drugs, policy and services, primary health, public health, rural health, statewide quality; and metropolitan health and aged care services division branches: acute, subacute and ambulance services) will be held during which the results of component 1 will be briefly presented, along with a short summary of the confirmed falls interventions that are contenders for implementation. A brain-storming activity will follow during which potential department policies and programmes will be identified. This group will also be asked for suggestions regarding relevant programmes of other government departments and non-government organisations for later consideration. Likely contenders include the Victorian Departments of Planning and Community Development and Transport., and the Commonwealth Department of Veterans' Affairs. The output from step 1 will be a list of potential policies and programmes for consideration.

The next step (step 2) will involve mapping the characteristics of the policies and programmes identified in step 1 and the characteristics of the confirmed falls interventions to identify characteristics of mutual relevance. A matrix will be prepared that maps the: specific target age group; other eligibility criteria; target health issue; and the main services provided against the policies and programmes. A similar matrix of the established falls interventions, with the most relevance to the subgroups identified in component 1 , will be prepared mapping the specific target age group, other eligibility criteria, and other health benefits of the intervention, against the key intervention components. The policies and programmes will be scored according to the match with each confirmed falls intervention. Scores of 2, 1 or 0 will be assigned if there is an exact or close to exact match, a partial match, or no match, respectively, with each dimension in the matrix. Departmental policies or programmes will then be ranked on this match score for each falls prevention intervention. The top ranked policies or programmes for each intervention will be selected for further analysis. This exercise will be undertaken for policies and programmes of the department, and highly relevant policies and programmes of other government departments and nongovernment organisations identified in step 1.

The final step (sep 3) will be a series of meetings with staff closely involved in the development and implementation of these polices and/or delivery of these short-listed programmes to identify mechanisms for integration of the established falls prevention strategies, and to identify factors that would facilitate, or be a barrier to, integration. For policies and programmes of other departments and organisations, meetings will be held with the relevant programme manager.

The output of component 3 will be the identification of policies and programmes with high potential for the integration of evidence-based falls interventions, along with some initial local facilitators for, and barriers to, integration. This material will feed into the development of the falls prevention and evaluation plans.

\section{Research component 4: facilitators of incorporation of falls prevention strategies into agency work programmes} Rationale

Limited evidence from the research literature, and our previous work, provide some direction for agencies to maximise the likelihood of sustainable implementation of falls interventions. ${ }^{45-51}$ Shediac-Rizkillah and Bone ${ }^{49}$ identify three critical influences on the potential sustainability of a communitybased health initiative. These are: (1) project design and implementation factors; (2) factors within the agency setting; and (3) factors in the broader community environment. They also highlight that in the majority of projects, sustainability is a 'latent' outcome that may or may not be achieved, and one that is often not effectively planned. Specific facilitators and barriers to implementation and sustainability within the community setting identified in our previous work are consistent with these main critical influences. Key barriers to sustained falls interventions included staff turnover and limited opportunities for ongoing training for staff (new and existing), and difficulties with engaging key local stakeholders. Facilitators included one or more 'champions', strong management support, falls intervention responsibilities included in clearly defined core roles for key staff, a desire to invest in longer term sustainability and linking with other healthy ageing messages. In addition, a strong cross-agency support network for key staff is essential for sharing their successes and challenges, and minimising duplication. $^{45}$

Resources have been directed into falls prevention activities in Australia by state and national governments for more than 10 years through key agencies including health services, PCP, local councils, divisions of general practice and others. However, most often such resources have been for time-limited projects and it has been difficult to embed evidence-based falls prevention interventions into long-term sustainable programmes of activities within the delivery agencies. The aims of this component are to: develop best practice sustainability guidelines for agencies that implement community-based falls interventions, and evaluate the feasibility of these guidelines.

\section{Methods}

Based on the existing research, guidelines for the sustained implementation of falls interventions will be developed, with recommendations, tips on implementation and indicators for achievement. A training programme will be developed to support agencies to use the guidelines in practice. The guidelines and the training programme will be trialled in eight departmental regions using PCP/community agencies (including home and community care, divisions of general practice, community health centres and others) that implement at least one of the identified most promising falls interventions. When possible, agencies selected for participation will include those in which the department has some level of responsibility. Participating agency staff will rate the feasibility of each element of the guidelines and recommend improvements. Each agency will be asked to review their current falls interventions in the context of the guidelines, and develop strategies and a work plan to address any identified gaps. The agency will be asked to implement this work plan if possible. In those agencies when this is possible, focus groups will be conducted 4 months later to determine achievements, facilitators and barriers to embedding changes within the organisation. Focus groups will involve several staff from each organisation, including at least one senior manager and one person with direct involvement in the falls intervention. Focus groups will be taped and professionally transcribed. The project officer will facilitate these discussions using a semistructured discussion guide. Thematic analysis will be undertaken independently by two experienced members of the research team. The output of this component will be best practice guidelines and associated resources to support agencies 
to adopt a more sustainable approach to falls interventions. The guidelines will have been developed, tested and refined and will be available for incorporation in the falls prevention plan.

\section{FALLS PREVENTION PLAN}

The 2-year falls prevention plan will be developed using the REAIM framework. This framework draws from the concepts of diffusion of innovation and the PRECEDE-PROCEED model, and ensures that five dimensions considered important for public health impact are systematically considered during programme planning. ${ }^{24}$ The research components will have identified department policies and programmes and those of associated external agencies, in which there is the greatest opportunity to impact falls significantly among older people by effectively implementing evidence-based interventions. The relevant evidence-based falls interventions for the two or three most promising of these programmes will be implemented. Strategies for the implementation of each intervention will be developed using the RE-AIM framework and are informed by the earlier research results. Table 1 shows the relationship between the four research components and the five RE-AIM factors as these relate to programme planning. There is no discrete research component for efficacy, because a strong body of evidence already exists regarding the falls interventions to be implemented. ${ }^{7}$ The challenge of translating research into policy, practice and the falls prevention plan will be addressed through a joint lead role involving the key departmental representatives of the partnership and the research team. This lead role will be supported by a working group composed of representatives from key stakeholder groups, such as departmental regions, PCP and service agencies. The working group will provide the lead role with advice on intervention planning, implementation and evaluation in real-world settings.

\section{EVALUATION PLAN}

The evaluation of the falls prevention plan will be designed as an integrated aspect of the plan, using the RE-AIM framework. Table 2 provides the definitions of each of the five RE-AIM factors as these relate to evaluation and shows examples of measures that might be considered using the integration of professionally prescribed home hazard assessment and modification into acute hospital discharge planning for high falls risk patients as an illustrative example only. In this example, it is assumed that this would be tested in a demonstration project in one or two hospitals. The specific measures relevant to the implementation of an intervention will depend on the nature of the intervention, the population being targeted and the implementation setting and context. It is likely that a number of different evidence-based falls interventions will be implemented within two or three existing policy or programme areas of the department and possibly by associated external agencies. In addition to the evaluation of the implementation of each falls intervention, trends in fall-related hospital admissions (including total bed days) will be monitored using specific key indicators. Implementation of the falls prevention plan will occur in years 2 and 3 , while the evaluation of the implementation will start in year 2 and continue into year 4 .

\section{Influence on health and research policy}

The National Injury Prevention and Safety Promotion Plan 2004-14, endorsed by the Australian health ministers, identifies falls prevention among older people as a priority. The most recent estimate of the direct cost of hospital treatment for falls among older people in Australia was A\$566 million for 2003-4. ${ }^{2}$ These costs will increase with the predicted threefold increase in the number of falls-related admissions in the next 50 years. ${ }^{14}$ Such an increase is unlikely to be accommodated by the capacity of the current hospital system, requiring over three-quarters of a million additional bed-days per year and 3320 nursing home places in 2051. ${ }^{14}$ Action to prevent falls among older people, to improve the health and independence of older Australians, and to relieve pressure on the already strained acute healthcare and rehabilitation sectors, is clearly needed. This challenge requires commitment at all levels of government.

The Victorian Department of Health plans, funds and delivers health, community and aged care services. It has four divisions (hospital and health service performance, mental health, drugs and regions, wellbeing, integrated care and ageing and strategy, policy and finance), each comprised a number of branches, which share the department's responsibilities for implementing policy and funding and monitoring service delivery.

Table 2 An illustration of the application of the RE-AIM framework for evaluating integration of home hazard assessment and modification into acute hospital discharge planning for high falls risk patients*

\begin{tabular}{|c|c|c|}
\hline RE-AIM factor & Definition & Example measures (method) \\
\hline Reach & $\begin{array}{l}\text { Absolute number, proportion and representativeness } \\
\text { of individuals who participate in the intervention }\end{array}$ & $\begin{array}{l}\text { Percentage of people over } 65 \text { years who are referred for occupational therapy } \\
\text { assessment on hospital discharge (records) } \\
\text { Comparison of demographic and medical history of those who are and are not referred } \\
\text { (analysis of routinely collected data) }\end{array}$ \\
\hline Efficacy & $\begin{array}{l}\text { Impact on important outcomes including potential } \\
\text { negative effects, quality of life and costs }\end{array}$ & $\begin{array}{l}\text { Percentage of people who actually receive an assessment (records) } \\
\text { Percentage of those for whom a home and/or behavioural modification is recommended } \\
\text { who actually make this change (survey of sample) } \\
\text { Impact on daily function for those who make a change (survey of sample) } \\
\text { Tracking of costs of intervention delivery (records) }\end{array}$ \\
\hline Adoption & $\begin{array}{l}\text { Proportion and representativeness of settings that } \\
\text { adopt the intervention }\end{array}$ & $\begin{array}{l}\text { Proportion of relevant staff aware of the policy and making the referrals (survey of staff) } \\
\text { Proportion of hospital wards that conduct routine referral (survey of all hospital wards) } \\
\text { Comparison of wards that do and do not conduct routine referral (analysis of routinely } \\
\text { collected data) } \\
\text { Barriers to adoption (key informant interviews) }\end{array}$ \\
\hline Implementation & Extent to which programme is delivered as intended & $\begin{array}{l}\text { Extent to which occupational therapists use standardised assessment tool (survey) } \\
\text { Length of time between discharge and assessment (records) }\end{array}$ \\
\hline Maintenance & $\begin{array}{l}\text { Extent to which intervention becomes part of routine } \\
\text { organisational practice } \\
\text { Long-term effects on outcomes six or more months } \\
\text { after the most recent intervention contact }\end{array}$ & $\begin{array}{l}\text { Proportion of hospital wards continuing to refer routinely (survey of hospital } \\
\text { wards } 6 \text { months after policy implemented) } \\
\text { Barriers to ongoing routine referral (key informant interviews) } \\
\text { Extent to which older people reverse the home modifications, and behavioural } \\
\text { modifications are maintained (survey } 6 \text { months after assessment) }\end{array}$ \\
\hline
\end{tabular}


The Department of Human Services is responsible for a range of other programmes and services including housing and community building. Aged care service providers are funded by the government to work in partnership with, and improve communication between, all stakeholders, and share information to create consumer and carer-friendly access to services, focus on healthy lifestyles, social connectedness and positive ageing. In 1995, the department's aged care branch established the falls prevention programme, located within the service development unit. Each financial year, a recommendation is made to the Minister for Senior Victorians regarding the way in which the falls prevention budget is allocated. As the ACB has lead responsibility for planning and funding falls interventions for older people in Victoria, it makes links to other relevant departmental programme areas, including the PCP strategy, home and community care, and ambulatory and continuing care services. Therefore, in addition to providing direct funding for specific falls interventions, the ACB falls prevention programme acts as a focal point, being well positioned to facilitate the coordination and integration of falls interventions across the department.

This project has been constructed to meet the strategic needs of the ACB, and as the falls prevention programme officer and manager are integral members of the partnership team, it clearly has the potential to inform falls prevention policy and decisionmaking regarding falls interventions directly under the auspice of the ACB.

The department has a record of effecting changes that clearly lead to better health outcomes as demonstrated by the following two examples: (1) The PCP strategy, commencing in 2000, has led to many improvements in service delivery, including decreased waiting times for appointments with diabetes educators due to improved referral networks, and a $50 \%$ reduction in time taken for patient registration thereby releasing health services staff time to focus on service delivery. A particularly exciting development is a partnership between the PCP and the Department of Justice, which has resulted in the integration of all gambler's help services into the PCP networks, thereby linking their clients into relevant health services and providing a coordinated prevention service. (2) The department has also demonstrated a capacity to overcome long-standing barriers interfering with access by Aboriginal communities to mainstream

\section{What is already known on the subject}

- Falls can be prevented among community-dwelling older people.

- Increasing rates of fall-related hospital admissions among community-dwelling older people in Victoria, Australia, suggests that the substantial intervention efficacy evidence base has not been fully translated to policy and practice.

\section{What this study adds}

- Improvements in knowledge required to bridge the gap between evidence from randomised trials and successful uptake of interventions.

- Contribution to knowledge regarding translation and implementation models. health services through the establishment of the strengthening home and community care in aboriginal communities strategy.

In addition to contributing to falls policy and decisionmaking, this project will lead to improvements in knowledge required to bridge the gap between evidence from randomised trials and the actual delivery of interventions based on this evidence. Given that the uptake of falls interventions by older people is required to achieve falls reductions, understanding the acceptability of confirmed falls interventions to older people is critical for planning programme delivery. Research on this topic is quite limited, especially in Australian populations. There is intense interest in the translation of evidence-based falls interventions into sustainable practice to achieve health gains. ${ }^{21} 22$ This project will contribute significantly to the body of knowledge regarding translation and implementation models.

Acknowledgements The authors are grateful to Alex Donaldson, Erin Cassell, Melissa Russell and Leon Flicker for their valuable comments on the draft protocol.

Funding This study was funded by a partnership project grant (ID 546282) from the Australian government National Health and Medical Research Council (NHMRC), and partnership funding from the Victorian Department of Health. CFF is supported by an NHMRC principal research fellowship (ID 565900)

\section{Competing interests None.}

Ethics approval Research components 1-3 have been approved by the Monash University Human Research Ethics Committee. Research component 4 will be subject to approval by the Latrobe University Human Research Ethics Committee.

Contributors All authors contributed to the conception and design, drafting of the article and approval of the final version.

Provenance and peer review Not commissioned; externally peer reviewed.

\section{REFERENCES}

1. Pointer S, Harrison J, Bradley C. National injury prevention plan priorities for 2004 and beyond: discussion paper. Adelaide, Australia: Australian Institute of Health and Welfare (AlHW), 2003.

2. Bradley C, Harrison J. Hospitalisations due to falls among older people, Australia, 2003-2004 Australian Institute of Health and Welfare (AIHW) Cat No INJCAT 96. Adelaide, Australia: AlHW, 2007.

3. Hindmarsh D, Hayen A, Finch C, et al. Relative survival after hospitalisation for hip fracture in older people in NSW, Australia. Osteoporos Int 2009;20:221-9.

4. Lord S, Sherrington C, Menz H. Falls in older people: risk factors and strategies for prevention. Sydney, Australia: Cambridge University Press, 2001.

5. Swift CG. Care of older people: falls in late life and their consequences-implementing effective services. BMJ 2001:322:855-7.

6. Campbell AJ, Borrie MJ, Spears GF, et al. Circumstances and consequences of falls experienced by a community population 70 years and over during a prospective study. Age Ageing 1990;19:136-41.

7. Gillespie LD, Robertson MC, Gillespie WJ, et al. Interventions for preventing falls in older people living in the community. Cochrane Database Syst Rev 2009;(2) CD007146. doi:10.1002/14651858.CD007146.pub2.

8. Kiel DP, O'Sullivan P, Teno JM, et al. Health care utilization and functional status in the aged following a fall. Med Care 1991:29:221-8.

9. Tinetti ME, Williams CS. The effect of falls and fall injuries on functioning in community-dwelling older persons. J Gerontol A Biol Sci Med Sci 1998;53:M112-19.

10. Cumming RG. Salkeld G, Thomas $M$, et al. Prospective study of the impact of fear of falling on activities of daily living, SF-36 scores, and nursing home admission. J Gerontol A Biol Sci Med Sci 2000;55:M299-305.

11. Salkeld G, Cameron ID, Cumming RG, et al. Quality of life related to fear of falling and hip fracture in older women: a time trade off study. BMJ 2000;320:341-6.

12. Australian Bureau of Statistics (ABS). Projections Australia, 2006-2101. Cat No 3220.0. Canberra, Australia: ABS, 2008.

13. Begg S, Vos T, Barker B, et al. The burden of disease and injury in Australia 2003 Australian Institute of Health and Welfare (AIHW) Cat No PHE 82. Canberra, Australia: AlHW, 2007

14. Moller J. Projected costs of fall related injury to older persons due to demographic change in Australia. Report to the Commonwealth Department of Health and Ageing under the National Falls Prevention for Older People Initiative. Canberra, Australia: Commonwealth of Australia, 2003.

15. Clapperton A, Cassell E. Preventing injuries in Victorian seniors 65 years and older Hazard, Edition No. 67. Melbourne, Australia: Monash University Accident Research Centre, 2008.

16. Boufous S, Finch C, Lord S. Incidence of hip fractures in New South Wales: are our efforts having an effect? Med J Aust 2004;180:623-6. 
17. Boufous $\mathbf{S}$, Finch $\mathrm{C}$, Lord $\mathrm{S}$, et al. The increasing burden of pelvic fractures in older people, New South Wales, Australia. Injury 2005;36:1323-9.

18. Day L, Finch CF, Harrison J, et al. Modelling the population level impact of Tai-Chi on falls and fall related injury among community dwelling older people. Inj Prev 2010;16:321-6.

19. Yardley L, Beyer N, Hauer K, et al. Recommendations for promoting the engagement of older people in activities to prevent falls. Qual Saf Health Care 2007; 16:230-4.

20. Yardley L, Kirby S, Ben-Shlomo Y, et al. How likely are older people to take up different falls prevention activities? Prev Med 2008;47:554-8.

21. Ganz DA, Alkema GE, Wu S. It takes a village to prevent falls: reconceptualizing fall prevention and management for older adults. Inj Prev 2008;14:266-71.

22. Robitaille $\mathbf{Y}$, Gauvin L. Fall prevention in older adults: towards an integrated population-based perspective. Inj Prev 2008;14:147-8.

23. Clemson L, Finch CF, Hill KD, et al. Fall prevention in Australia: policies and activities. Clin Geriatr Med 2010;26:733-49.

24. Glasgow RE, Vogt RM, Boles SM. Evaluating the public health impact of health promotion interventions: the RE-AIM framework. Am J Public Health 1999;89:1322-7.

25. Mathers CD, Murray CJL, Ezzati M, et al. Population health metrics: crucial inputs to the development of evidence for health policy. Popul Health Metr 2003;1:6.

26. Boufous S, Finch C, Close J, et al. Hospital admissions following presentations to emergency departments for a fracture in older people. Inj Prev 2007:13:211-14.

27. Camilloni L, Farchi S, Giorgi Rossi P, et al. Mortality in elderly injured patients: the role of comorbidities. Int J Inj Contr Saf Promot 2008;15:25-31.

28. Boufous S, Finch C. Estimating the incidence of hospitalised injurious falls: impact of varying case definitions. Inj Prev 2005;11:334-6.

29. Sundararajan V, Henderson T, Perry $C$, et al. New ICD-10 version of the Charlson comorbidity index predicted in-hospital mortality. J Clin Epidemiol 2004;57:1288-94.

30. Finch C, Boufous S. Do inadequacies in ICD-10 activity coded data lead to underestimates of the population frequency of sports injuries? Inj Prev 2008;14:202-4.

31. Turner R, Hayen A, Dunsmuir W, et al. Spatial temporal modelling of hospitalisations for fall-related hip fracture in older people. Osteoporos Int 2009;20:1479-85.

32. Byers AL, Allore H, Gill TM, et al. Application of negative binomial modelling for discrete outcomes: a case study in aging research. J Clin Epidemiol 2003;56:559-64.

33. Yardley L, Bishop Fl, Beyer N, et al. Older people's views of falls-prevention interventions in six European countries. Gerontologist 2006:46:650-60.

34. Commonwealth of Australia. National falls prevention for older people initiative "Step Out with Confidence": a study into the information needs and perceptions of older Australians concerning falls and their prevention. Pub Approval No 2848. Canberra, Australia: Commonwealth of Australia, 2001.
35. Ballinger C, Clemson L. Older people's views about community falls prevention: an Australian perspective. Br J Occup Ther 2006;69:263-70.

36. Whitehead CH, Wundke CR, Crotty M. Attitudes to falls and injury prevention: what are the barriers to implementing falls prevention strategies. Clin Rehabil 2006;20:536-42

37. Snodgras S, Rivett DA, Mackenzie L. Perceptions of older people about falls prevention and physical activity. Aust J Ageing 2005;24:114-18.

38. Nutbeam D, Harris E. Theory in a nutshell. Sydney: University of Sydney, 1998.

39. Rogers RW. Cognitive and physiological processes in fear appeals and attitude change: a revised theory of protection motivation. In: Cacioppo J, Petty R, eds. Social Psychophysiology. New York: Guilford Press, 1983.

40. Yardley L, Donovan-Hall M, Francis K, et al. Attitudes and beliefs that predict older people's intention to undertake strength and balance training. J Gerontol B Psychol Sci Soc Sci 2007;62:P119-25.

41. Hill AM, McPhail S, Hoffman T, et al. A randomized trial comparing digital video disc with written delivery of falls prevention education for older patients in hospital. J Am Geriatr Soc 2009:57:1458-63.

42. Shieh G, Jan S, Randle RH. Power and sample size determinations for the Wilcoxon signed-rank test. J Stat Comput Simul 2007;77:717-24.

43. Finch C, Day L, Donaldson A, et al. Determining policy-relevant formats for the presentation of falls research evidence. Health Policy 2009;93:207-13

44. Poulos R, Donaldson A, Finch C. Towards evidence informed sports safety policy - understanding the role of actors, context, and process in policy development. Inj Prev 2010;16:127-31.

45. Clemson L, Matthews M, Dean C, et al. Translating research into practice: sustainability of a community based falls prevention program in minority communities. Sydney, Australia: University of Sydney, 2008.

46. Baker D, Gottschalk M, Bianco LM. Step by step: integrating evidence-based fallsrisk management into senior centers. Gerontologist 2007;47:548-54.

47. Fortinsky R, Baker D, Gottschalk $M$, et al. Extent of implementation of evidencebased falls prevention practices for older patients in home health care. J Am Geriat Soc 2008; 56:737-43

48. Greenhalgh T, Robert G, Macfarlane F, et al. Diffusion of innovations in service organizations: systematic review and recommendations. Millbank 0 2004;82:581-629.

49. Shediac-Rizkallah $\mathbf{M}$, Bone L. Planning for the sustainability of health promotion programs: conceptual framework and future directions for research, policy and practice. Health Educ Res 1998;13:87-108.

50. Rubenstein L, Pugh J. Strategies for promoting organizational and practice change by advancing implementation research. J Gen Intern Med 2006;21(Suppl 2):S58-64

51. Deery HA, Day LM, Fildes BN. An impact evaluation of a falls prevention program among older people. Accid Anal Prev 2000;32:427-33. 